\title{
Attentional Modulation of Firing Rate Varies with Burstiness across Putative Pyramidal Neurons in Macaque Visual Area V4
}

\author{
Emily B. Anderson, Jude F. Mitchell, and John H. Reynolds \\ Systems Neurobiology Laboratory, The Salk Institute, La Jolla, California 92037
}

One of the most well established forms of attentional modulation is an increase in firing rate when attention is directed into the receptive field of a neuron. The degree of rate modulation, however, can vary considerably across individual neurons, especially among broad spiking neurons (putative pyramids). We asked whether this heterogeneity might be correlated with a neuronal response property that is used in intracellular recording studies to distinguish among distinct neuronal classes: the burstiness of the neuronal spike train. We first characterized the burst spiking behavior of visual area V4 neurons and found that this varies considerably across the population, but we did not find evidence for distinct classes of burst behavior. Burstiness did, however, vary more widely across the class of neurons that shows the greatest heterogeneity in attentional modulation, and within that class, burstiness helped account for differences in attentional modulation. Among these broad spiking neurons, rate modulation was primarily restricted to bursty neurons, which as a group showed a highly significant increase in firing rate with attention. Furthermore, every bursty broad spiking neuron whose firing rate was significantly modulated by attention exhibited an increase in firing rate. In contrast, non-bursty broad spiking neurons exhibited no net attentional modulation, and, although some individual neurons did show significant rate modulation, these were divided among neurons showing increases and decreases. These findings show that macaque area $\mathrm{V} 4$ shows a range of bursting behavior and that the heterogeneity of attentional modulation can be explained, in part, by variation in burstiness.

\section{Introduction}

The longest studied form of attention-dependent neuronal response modulation is a change in mean firing rate, which typically increases when attention is directed to a stimulus within the receptive field of a neuron (Treue, 2003; Reynolds and Chelazzi, 2004; Knudsen, 2007). However, the degree of attention-dependent firing rate modulation across individual neurons can vary considerably, with some neurons even showing statistically significant reductions in firing rate with attention. Recently, Mitchell et al. (2007) found that, in area V4, an intermediate stage of visual processing that is strongly modulated by attentional state, two classes of neurons can be distinguished: narrow spiking neurons (putative interneurons) and broad spiking neurons (putative pyramidal neurons). Much of the heterogeneity in attentional modulation was restricted to broad spiking neurons. We wondered whether other properties of the responses of the neurons, such as

Received Jan. 3, 2011; revised June 10, 2011; accepted June 11, 2011.

Author contributions: J.F.M. and J.H.R. designed research; E.B.A. and J.F.M. performed research; E.B.A. and J.F.M. analyzed data; E.B.A., J.F.M., and J.H.R. wrote the paper.

This work was supported in part by National Eye Institute Grant EY13802 (J.F.M. and J.H.R.), The Gatsby Charitable Foundation (J.H.R.), a National Institutes of Health Training Fellowship (J.F.M.), and National Science Foundation (NSF) Grant SBE 0542013 (to the Temporal Dynamics of Learning Center, an NSF Science of Learning (enter). We thank Kristy Sundberg for help in data collection, and two anonymous reviewers for helpful comments on an initial draft of this manuscript. We thank C. Williams and J. Reyes for help with animals and technical support. The authors declare no competing financial interests.

Correspondence should be addressed to John H. Reynolds, Systems Neurobiology Laboratory, The Salk Institute, 10010 Torrey Pines Road, La Jolla, CA 92037. E-mail: reynolds@salk.edu.

DOI:10.1523/JNEUROSCI.0027-11.2011

Copyright $\odot 2011$ the authors $\quad 0270-6474 / 11 / 3110983-10 \$ 15.00 / 0$ variation in their spiking statistics, could help account for this heterogeneity.

Several early studies concluded that the spiking of cortical neurons can, to a first approximation, be described as Poisson, with spike count variance proportional to the mean rate and spike timing that is nearly independent of preceding spike history (Tolhurst et al., 1983; Softky and Koch, 1993; Shadlen and Newsome, 1998). However, recent studies in the awake primate that have examined spiking statistics in more detail have revealed a diversity of firing patterns across neocortical regions (Maimon and Assad, 2009), as well as across neurons recorded within individual cortical areas (Bair et al., 1994; Friedman-Hill et al., 2000; Compte et al., 2003; Joelving et al., 2007; Katai et al., 2010). Indeed, many intracellular recording studies have used discharge patterns such as bursting to distinguish among different neocortical neuronal classes (McCormick et al., 1985; Nowak et al., 2003). These findings underscore the importance of examining the spiking statistics within a brain area of interest. They also raise the possibility that we may gain valuable insights from characterizing deviations from Poisson spiking within an area and examining whether these deviations correlate with other neuronal response properties.

With this motivation, we asked whether neurons in macaque visual area V4 exhibit deviations from Poisson-like behavior. We find that V4 neurons exhibit a broad continuum of spiking statistics, with some responding much like Poisson processes, whereas others exhibited strikingly bursty behavior. We next considered whether this diversity of discharge patterns might 


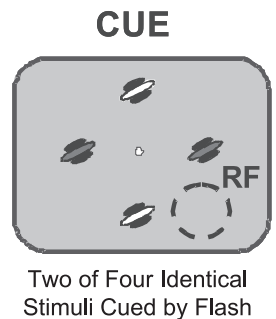

(500ms)

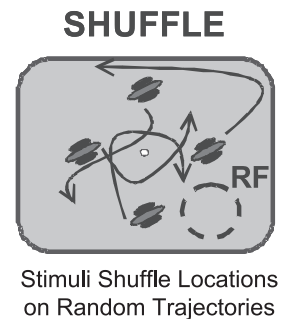

(950ms)

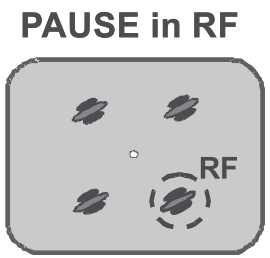

Stimuli Pause

$(1000 \mathrm{~ms})$
SHUFFLE

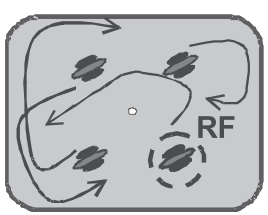

Stimuli Shuffle Locations on Random Trajectories (950ms)

\section{SACCADE}

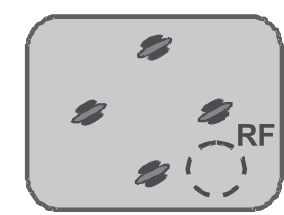

Fixation Point Off

Saccade to Targets

Figure 1. Attentional state was controlled with a multi-object tracking task. Animals initiated trials by fixating a central point. Four identical Gabor stimuli then appeared, and one or two of them were cued as targets with a brief elevation in luminance. The monkey then maintained fixation while attentively tracking the targets as they moved along independent randomized trajectories that brought one of the stimuli into the receptive field (RF), at which point all four stimuli paused for $1000 \mathrm{~ms}$. The stimuli then shuffled position a second time, with randomized trajectories that placed them at equally eccentric positions outside the receptive field. The fixation point then disappeared, indicating that the monkey should saccade to the previously cued targets. Juice reward was delivered if the monkey correctly made a saccade to each cued stimulus and none of the distracter stimuli.

correspond with different patterns of attentional modulation. We find that, among broad spiking neurons, differences in burstiness predict attentional rate modulation, with bursty broad spiking neurons showing more consistent increases in firing rate with attention than non-bursty broad spiking neurons.

\section{Materials and Methods}

Electrophysiology and receptive field characterization. All procedures were approved by The Salk Institute Institutional Animal Care and Use Committee and conformed to NIH guidelines for the humane care and use of animals in research. Monkeys were prepared for neuronal recording following procedures described by Mitchell et al. (2007). After training each animal on the behavioral task described below, recordings were made from two to five tungsten electrodes (FHC) that were advanced until action potentials of single neurons could be isolated based on action potential waveform shape. Neuronal signals were recorded extracellularly, filtered (Butterworth filter, six-pole, $3 \mathrm{~dB}$ cutoff at $154 \mathrm{~Hz}$ and 8.8 $\mathrm{kHz}$ ), and stored using the Multichannel Acquisition Processor system (Plexon). Spike waveforms crossing a negative threshold, which was set to exclude noise, were stored for later offline analysis. Units were identified as isolated in offline analysis (Offline Sorter; Plexon) if the first three principle components of their waveform shape formed a clearly separable cluster from noise and other units. After isolating one or more neurons, receptive fields were mapped using a subspace reverse correlation procedure (Ringach et al., 1997; Mitchell et al., 2007). In this procedure, colored Gabor stimuli (eight orientations, six colors, $80 \%$ luminance contrast, 1.2 cycles $/^{\circ}$, Gabor Gaussian half-width of $2^{\circ}$ ) were flashed at random locations (chosen from a grid with $3^{\circ}$ spacing) to determine a single stimulus location that would elicit a robust visual response. When multiple neurons were recorded simultaneously, the features and location of the stimulus were selected to excite the best isolated units.

Stimulus presentation and eye movement monitoring. Stimuli were presented on a computer monitor (Sony Trinitron Multiscan, TC, $640 \times 480$ pixel resolution, $120 \mathrm{~Hz}$ ) placed $57 \mathrm{~cm}$ from the eye. Experimental control was handled by NIMH Cortex software (http://www.cortex.salk.edu).

Eye position was continuously monitored with an infrared eye tracking system $(240 \mathrm{~Hz}$, ETL-400; ISCAN). We detected microsaccades as described by Mitchell et al. (2007). Matlab source code and example data are available online at (http://www.snl.salk.edu/ jude). Briefly, saccades were defined as points in the eye position traces in which a $400 \mathrm{~ms}$ window around the time point was better fit with a model with a saccade-like discontinuity than with a smooth polynomial spline function. After determining model parameters by minimizing the mean squared error, saccades were identified as points in time when the variance explained by the saccade model was $>30 \%$ greater than for the spline model, with additional constraint that the instantaneous velocity at that point exceeded $10 \%$, and the instantaneous acceleration exceeded $1000^{\circ} / \mathrm{s}^{2}$. Figure $5 \mathrm{~A}$ provides an example trial with raw eye position traces, model fits, and detected microsaccades.

Task and stimuli. Two monkeys performed a multiple-object tracking task (Fig. 1) that has been used to study attention in humans (Pylyshyn and Storm, 1988; Sears and Pylyshyn, 2000; Cavanagh and Alvarez, 2005) and nonhuman primates (Mitchell et al., 2007, 2009; Sundberg et al., 2009). The animals began each trial by fixating a central point and maintained fixation until the end of the trial. After $200 \mathrm{~ms}$, four identical Gabor stimuli appeared (40\% luminance contrast). The color and orientation of these stimuli were chosen based on the subspace reverse correlation map to produce a strong response. The positions of the stimuli were selected to fall at regular intervals along an invisible ring of equal eccentricity, selected such that all of the stimuli fell outside of the receptive fields of the neurons. One or two stimuli were then cued as targets by a brief elevation in luminance. All four stimuli then moved along independent, randomly generated trajectories that positioned the stimuli at four new, equally eccentric positions. This placed one of the stimuli at the center of the receptive field of the neuron and the others outside the receptive field. The trajectories were designed to match stimulation history across the two attention conditions, by using the identical trajectories in the attended and unattended trials, and by preventing all but one stimulus from entering the receptive field. The stimuli then paused for $1000 \mathrm{~ms}$ before moving to a final set of equally eccentric positions and stopping. At this point, the fixation point disappeared, signaling the animal to make a saccade to each cued target. To minimize the development of spatial biases, the starting and ending positions for the target and nontarget stimuli were symmetrically balanced. Correct identification of the targets resulted in a liquid reward. Only correctly completed trials with two of four stimuli tracked were analyzed.

Inclusion criteria. We recorded from 206 well-isolated neurons from two male monkeys ( $n=53$ for monkey B, $n=153$ for monkey M). We restricted our discharge pattern analyses to units whose response on trials when attention was directed away from the receptive field exceeded $5 \mathrm{~Hz}$, averaged over the final $500 \mathrm{~ms}$ of the stimulus pause period, and was significantly greater than the mean spontaneous firing rate averaged over the $250 \mathrm{~ms}$ preceding the onset of the Gabor stimuli (Mann-Whitney $U$ test, $p<0.05)$. This resulted in 84 neurons being excluded. In addition, four units were excluded because their waveforms did not have the typical biphasic shape, with a trough followed by a clearly defined peak, and they could not therefore be classified as narrow or broad spiking. In total, 118 neurons met these selection criteria. Unless otherwise specified, analysis of spiking statistics was restricted to the final $800 \mathrm{~ms}$ of the pause period (the "sustained period"), which excluded periods of transient response as stimuli entered or exited the receptive field, and thus the mean firing rate was relatively stationary.

Broad and narrow spiking classification. As described previously (Mitchell et al., 2007), we divided neurons into narrow and broad spiking subpopulations based on waveform duration (see Fig. 3). We defined waveform duration to be the time from the trough to the peak of the average waveform (Mitchell et al., 2007). We selected this metric on the basis of studies showing that this measure best distinguishes putative pyramidal neurons from putative fast-spiking interneurons in the neocortex (Barthó et al., 2004). The distribution of spike waveform duration was significantly bimodal across all isolated cells with biphasic spike 
waveforms ( $n=202$, Hartigan's dip test, $p<0.0001$ ) and also across the subset of these cells with significant visual responses $(n=118$, Hartigan's dip test, $p<0.01)$. Narrow and broad spiking neurons were separated based on the trough between the two modes of the waveform duration distribution, with narrow spiking neurons defined as those ranging in duration from 100 to $224 \mu \mathrm{s}$ and broad spiking neurons defined as those ranging in duration from 225 to $500 \mu \mathrm{s}$.

Burst analysis. To assess the degree of burstiness of a given neuron, we computed the burstiness/refractoriness index (B.R.I.), as defined by Compte et al. (2003), over the 800 ms sustained period. First, we calculated the autocorrelation function of the neuron separately for each attention condition. We then subtracted the shuffle predictor for that condition. The shuffle predictor is defined as the mean cross-correlation across all pairs of trials of an individual neuron and corresponds to the autocorrelation of a Poisson process with the same mean time course as the cell. By subtracting the predictor, we remove any trial-locked fluctuations in spiking that result from repeated presentation of the stimulus. After this subtraction, we normalized the result to the SD (standard deviation) of the shuffle predictor at each time lag. This results in a shuffle-corrected autocorrelation function that has been normalized for the SD in the shuffle predictor, for the attended and unattended conditions. The B.R.I. is defined to be the average height of the unattended shuffle-predictor-normalized autocorrelation function over the interval corresponding to 1-4 ms. This measure is expressed in units of the shuffle-predictor SDs. The logic of this metric is analogous to a $z$-transformation. The $z$-score indicates how many SDs an observation is from the population mean. By assuming a normal distribution with a given mean and SD, one can determine whether the observation falls outside the range expected by chance. The metric used here to assess burstiness assumes a Poisson distribution, which, by definition, is not bursty, because the occurrence of each spike is independent of spiking history. For analyses in which we divided the units into bursty and nonbursty populations (see Fig. $4 A-D$ ), we define neurons as bursty if their B.R.I. exceeded two (central autocorrelation peak $>2$ SDs above the shuffle predictor). Values greater than two indicate that the neuron exhibits more short-duration interspike intervals (ISIs) than would be expected by chance from a rate-matched Poisson process, indicating that the neuron is bursty. Large negative values indicate an extended period of refractoriness.

Because we observe a continuum of discharge patterns across our data (see Fig. 3), this division is not intended to imply that these are intrinsic subclasses of neurons but rather to illustrate the differences we observe between significantly bursty and nonsignificantly bursty neurons. We obtained similar results when we used a B.R.I. threshold of 1 SD to define bursty and non-bursty cells.

\section{Results}

We characterized the discharge patterns of 118 visually driven neurons in area $\mathrm{V} 4$, in two macaques as they performed the attention-demanding tracking task depicted in Figure 1. This task allowed us to direct attention toward or away from a stimulus that we positioned within the receptive fields of the neurons. We then asked whether there was a relationship between attentional modulation and the discharge patterns we observed in broad and narrow spiking neurons.

\section{Assessment of discharge patterns}

The population of V4 neurons exhibited a wide range of discharge patterns in response to sustained stimuli, ranging from highly bursty neurons that frequently fired doublets and triplets, to neurons with long relative refractory periods that rarely, if ever, exhibited bursts. Example neurons are presented in Figure 2 , which also serves to illustrate several different ways of visualizing burstiness. The neuron in Figure $2 \mathrm{~A}$ is a broad spiking neuron (see mean waveform in top right) that frequently fired in bursts. The top left shows $100 \mathrm{~ms}$ samples of the spiking behavior of this neuron, taken from unattended trials. In this spike raster, it can be seen that the neuron often fired several spikes in close succession. Another way to visualize the bursting behavior of neurons is through their ISI return map, which plots each spike according to the ISI before and after each spike. As shown in the middle, the ISI return map reveals clusters of spikes. Points in the bottom right corner correspond to spikes at the beginning of bursts, whereas points in the top left corner correspond to spikes at the end of a burst. Points in the bottom left corner correspond to spikes in the middle of bursts. Burstiness can also be revealed by looking at the ISI distribution, shown in the top right. For a unit with Poisson firing, the ISI distribution would appear as an exponential distribution decaying from the shortest ISI. Here we can see a strong peak in the ISI distribution at short intervals of 2-4 ms indicating bursting behavior. The bottom right shows the normalized spike autocorrelation function of the neuron in the unattended condition, which is arguably a simpler way of looking at how spiking deviates from Poisson behavior. It plots the probability of firing at a given delay, normalized by its deviation from the expectation of a rate-matched Poisson process (see Materials and Methods). For a Poisson processes, this normalized autocorrelation function would appear as a flat line fixed at zero. Similar to the early peak seen in the ISI distribution, we see once again that this neuron exhibits a strong early peak centered near $2-4$ ms. Unlike the ISI distribution, however, this normalized autocorrelation function makes it clear that this peak deviates significantly from the Poisson expectation, indicating that the spikes following at short delays are occurring much more frequently than would be expected for a rate-matched Poisson process.

The degree of burst spiking varied considerably over the population, as can be seen by comparing the example neurons in Figure 2. The neurons in Figure 2, $B$ (broad spiking) and $D$ (narrow spiking), showed relatively modest deviations from Poisson spiking. This can be seen by a lack of clustering in the ISI return map (middle column) and a normalized autocorrelation function that deviated only modestly from the Poisson expectation (horizontal dashed line), with a dip at short ISIs corresponding to a period of relative refractoriness. Some narrow spiking neurons also exhibited burst firing, as seen for the example neuron in Figure $2 C$, although this was less common.

We used the B.R.I. introduced by Compte et al. (2003) to quantify the distribution of burst firing behavior across the population. This metric examines the deviation of the first $4 \mathrm{~ms}$ of the autocorrelation function of a unit from that of its shufflepredictor (see Materials and Methods). A positive B.R.I. indicates that the neuron tended to fire spikes in bursts, as indicated by a larger number of closely spaced spikes than would be expected from a rate-matched Poisson process. In contrast, a negative value indicates an extended relative refractory period. Using this metric, we find that both narrow and broad spiking cells exhibit a continuum of discharge patterns, although broad spiking neurons exhibit more extreme burstiness. Figure 3 displays the distribution of B.R.I. as a function of spike waveform duration across the entire population. Those neurons with statistically significant burst firing (B.R.I. $>2$; see Materials and Methods) are indicated by dark gray circles and non-bursty neurons are indicated by light gray circles. There was a wide distribution of burst firing across the population, but it was not clearly bimodal (Hartigan's dip test, $p>0.5$, histogram not shown). However, as shown previously (Mitchell et al., 2007), there was a bimodal distribution of spike waveforms as indicated by the histogram at the bottom of the figure (Hartigan's dip test, $p<0.001$ ). We therefore divided neurons into narrow and broad spiking categories based on this histogram, with narrow spiking defined as those 
units with waveform durations $<225 \mu \mathrm{s}$ (vertical line), and examined burstiness separately for each class. Narrow spiking neurons tended to have lower B.R.I.s, indicating a more modest tendency to fire action potentials in bursts. The broad spiking population showed a wider range of values, including some neurons that were extremely bursty. This difference in burstiness across narrow and broad spiking neurons was highly significant (MannWhitney $U$ test, $p<0.000005$; narrow median B.R.I. of -0.87 ; broad median B.R.I. of 2.67).

\section{Relationship between burst firing and} attention-dependent rate modulation

Broad spiking neurons exhibit more heterogeneous attentional rate modulation than narrow spiking neurons (Mitchell et al., 2007). The present finding, that they also show more variability in their spiking statistics, led us to wonder whether the two phenomena could be related. To examine this, we asked whether the degree of burstiness corresponds to the magnitude of attentional modulation. We first subdivided the narrow and broad spiking populations into bursty and non-bursty groups according to whether the B.R.I.s of the neurons exceeded 2 SDs of the B.R.I.s of Poisson processes matched in mean firing rate (see Materials and Methods). The left column of Figure 4 shows mean population responses of these groups to attended (red) and unattended (blue) stimuli. Remarkably, one group of neurons, non-bursty broad spiking neurons, exhibited nearly identical mean firing rates across attention conditions, indicating that, on average, these neurons are not modulated by attention (median unattended, $11.80 \mathrm{~Hz}$; median attended, 11.95 $\mathrm{Hz}$; median $2.80 \%$ increase in absolute firing rate; Wilcoxon's signed-rank test, $p>0.3)$. The other three groups of neurons exhibited significant attention-dependent increases in firing rate (broad spiking bursty neurons: median unattended, $10.26 \mathrm{~Hz}$; median attended, $13.79 \mathrm{~Hz}$; a median $21.72 \%$ increase; Wilcoxon's signed-rank test, $p<0.00001$; narrow spiking bursty neurons: median unattended, $18.05 \mathrm{~Hz}$; median attended, $27.81 \mathrm{~Hz} ; 25.40 \%$; $p<0.01$; narrow spiking non-bursty neurons: median unattended, $18.00 \mathrm{~Hz}$; median attended, $24.80 \mathrm{~Hz}$; $26.72 \%$; $p<0.000005)$.

To examine the distribution of attentional modulation of firing rate across neurons in each subpopulation, we computed a normalized attention index (rate A.I.) for each neuron: (attended mean rate - unattended mean rate)/(attended mean rate + unattended mean rate). Positive values of this index correspond to neurons showing attention-dependent increases in rate, and negative values correspond to decreases. The distribution of this index, for each group, appears in Figure 4 (middle column). Black
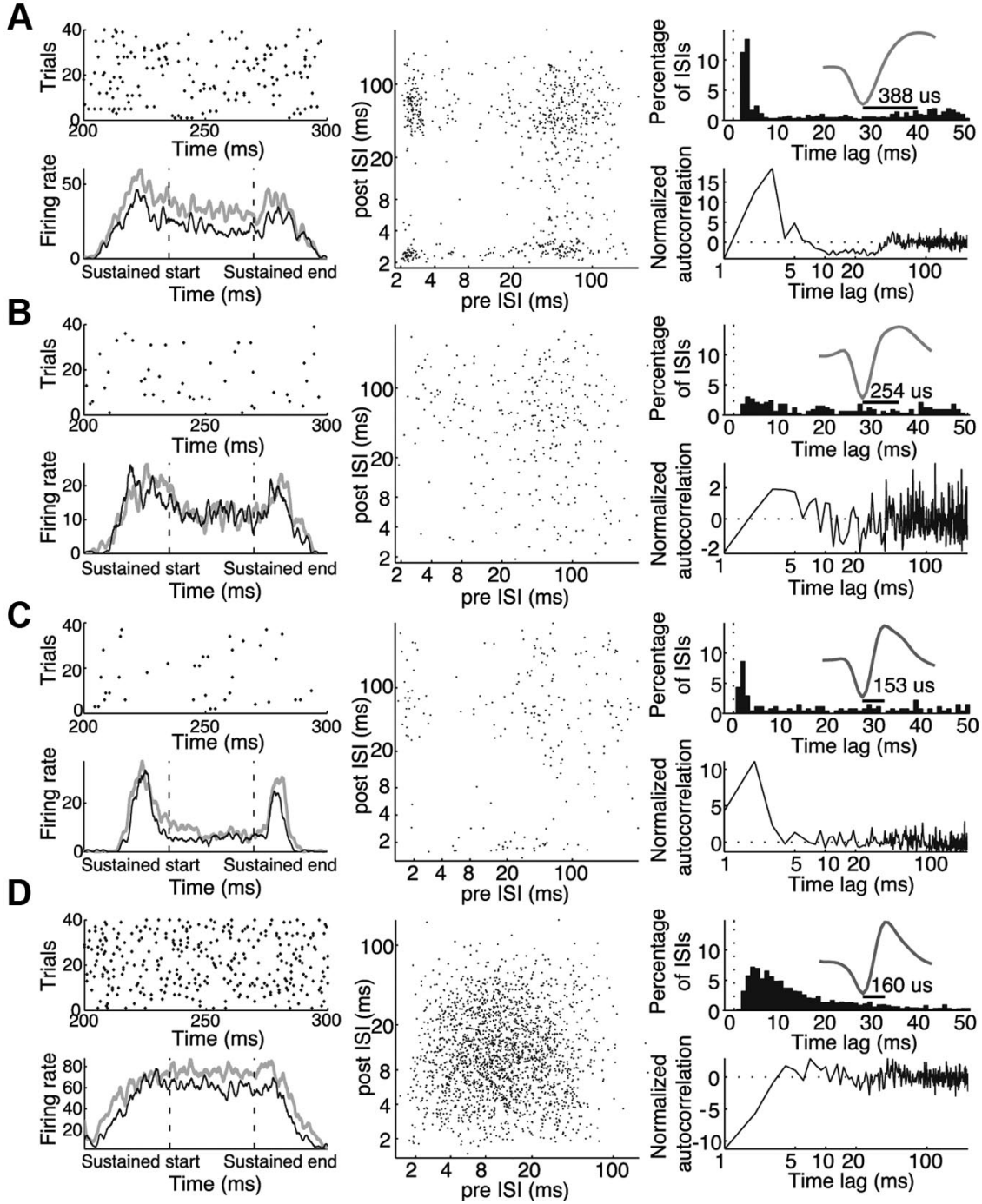

Figure 2. Examples of four individual neurons with varying degrees of burstiness. $A$, Example of a bursty broad spiking neuron $\boldsymbol{B}$, Example of non-bursty broad spiking neuron. $\boldsymbol{C}$, Example of a bursty narrow spiking neuron. $\boldsymbol{D}$, Example of a non-bursty narrow spiking neuron. For each neuron, top left panels show spike raster plots of a $100 \mathrm{~ms}$ window within the sustained period for the first 40 correct unattended trials. Bottom left panels show the neuronal response (spikes per second) averaged across trials, to targets (gray) and distracters (black) as they entered the receptive field, paused, and exited the receptive field. These response time courses were smoothed by convolving each with a Gaussian kernel ( $\sigma=25 \mathrm{~ms}$ ). Middle panels show interspike interval return (he normalized autocorrelation functions (autocorrelation minus the shuffle predictor divided by the SD of the shuffle predictor; see Materials and Methods) for the unattended condition. Dashed line at 0 indicates the normalized autocorrelation function of a rate-matched Poisson process.

bars indicate individual neurons that showed statistically significant modulation of firing rate (Mann-Whitney $U$ test, $p<$ $0.001)$. Consistent with the above population median and mean modulation, we find that, among broad spiking neurons, attention-dependent increases in rate are primarily restricted to bursty cells. Among broad spiking bursty neurons, all neurons with significant attention-dependent rate modulation showed increases in firing rate when attention is directed into the receptive field of the neuron ( $n=12$ of 40 neurons, 30.0\%). Similarly, all narrow spiking neurons with significant attentional rate modulation showed increases in firing rate (bursty narrow, $n=7$ of 13 neurons, 53.9\%; non-bursty narrow, $n=15$ of 34 neurons, $44.1 \%)$. For all three of these groups, the distributions of A.I.s 

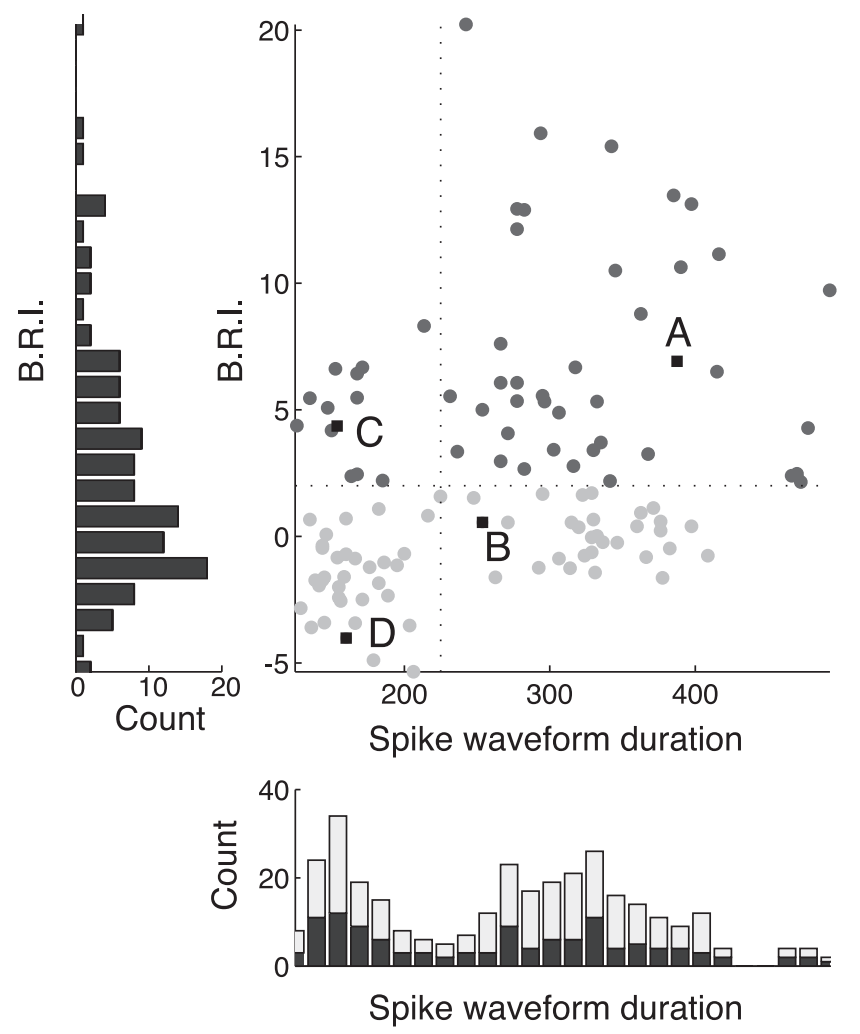

Figure 3. Population scatter plot of spike waveform duration (microseconds) versus B.R.I. $\boldsymbol{A}-\boldsymbol{D}$ indicate example units from Figure 2. Dark gray circles correspond to bursty neurons, defined as neurons whose burstiness exceeded 2 SDs of a rate-matched Poisson process (B.R.I. $>2$ ). Light gray circles correspond to non-bursty cells. The left panel shows the distribution of the B.R.I. across the population, which was not significantly bimodal. The bottom panel shows the distribution of spike waveform durations, which is significantly bimodal both for visually driven cells (dark bars; Hartigan's dip test, $p<0.01$ ) and the entire population (light bars indicate nonvisually driven neurons; Hartigan's dip test, $p<0.0001$ ).

showed significant increases, according to a Wilcoxon's signedrank test (bursty broad, $p<0.00005$; bursty narrow, $p<0.005$; non-bursty narrow, $p<0.00001)$. The distributions of the rate A.I. did not differ significantly among these three groups. This contrasts markedly with the non-bursty broad spiking group, in which attentional modulation was less common and more heterogeneous, with similar numbers of increases and decreases in firing rate observed (three decreases and five increases of 31 neurons, 9.7 and 16.1\%). Furthermore, the distribution of A.I.s for the non-bursty broad spiking group differed significantly from each of the other three groups (Mann-Whitney $U$ test, bursty broad, $p<0.006$; bursty narrow, $p<0.05$; non-bursty narrow, $p<0.0005)$.

Because the degree of burstiness appears to fall along a continuum and not into a bimodal distribution, one possible concern is that the above findings depended on the threshold we set to divide neurons into bursty and non-bursty groups (B.R.I. $>2$ ). Therefore, we also asked whether there was a correlation between the degree of burstiness and the strength of attentional mean rate modulation. We computed the correlation between the B.R.I. and the rate A.I. The results are shown in Figure 4, E and F, which shows population scatter plots of B.R.I. versus rate A.I. for the narrow $(E)$ and broad $(F)$ spiking populations. Within the narrow spiking subpopulation, we observe increases in firing rate with attention regardless of the degree of burstiness, with all significantly modulated neurons showing increases in firing rate with attention. We also observe no correlation between rate A.I. and B.R.I. among narrow spiking neurons (Fig. $4 E$ ) (Spearman's nonparametric correlation, $\left.p>0.7, R_{\mathrm{s}}=0.0490\right)$. In contrast, we find that, among broad spiking neurons, there is a significant correlation between greater degrees of burstiness and greater attention-dependent increases in firing rate (Fig. $4 F$ ) (Spearman's rank correlation, $p<0.05, R_{\mathrm{s}}=0.2499$ ). This suggests that the differences in attentional modulation we observe between the bursty and non-bursty broad spiking groups are not an artifact of our choice of threshold but rather reflect a relationship between burstiness and attention-dependent modulations of firing rate. A remaining concern was that this correlation could potentially be driven by outliers. To test this, we measured correlation among broad spiking neurons after excluding neurons with rate A.I. or B.R.I. values that exceeded 1.5 SDs of the broad spiking population mean. After excluding these neurons, the correlation remained (Fig. $4 F$, filled circles within dashed orange region) $\left(p<0.01, R_{\mathrm{s}}=0.3583\right)$. Thus, we conclude that, among broad but not among narrow spiking neurons, attention-dependent increases in firing rate are observed primarily among bursty neurons.

\section{Differences in firing rate do not explain attention differences among broad spiking neurons}

Another possible concern is that this difference in rate A.I. could be attributable to differences in firing rate across the broad spiking groups. If the non-bursty broad spiking group had lower firing rates than the bursty broad spiking group, this could reduce statistical power, impairing our ability to detect attentional modulation rather than reflecting an actual difference in attentional rate modulation between these two groups. To test this, we compared the unattended firing rate distributions of the bursty and non-bursty broad spiking groups. We find that there is no significant difference in the unattended firing rates between the nonbursty and bursty broad spiking neurons (Mann-Whitney $U$ test, $p>0.8$; bursty broad median, $10.26 \mathrm{~Hz}$; non-bursty broad median, $11.80 \mathrm{~Hz}$ ), nor is there a significant difference in the absolute number of action potentials recorded in the unattended state for the two populations of neurons (Mann-Whitney $U$ test, $p>$ 0.6 ; bursty broad median, 353.5 spikes per neuron; non-bursty broad median, 378 spikes per neuron).

To examine this further, we asked whether mean firing rate in the unattended condition correlated with attentional modulation. There is no correlation between mean unattended firing rate and rate A.I. for either the broad spiking neurons (Spearman's rank correlation, $\left.p>0.4, R_{\mathrm{s}}=-0.0917\right)$ or across the entire population (Spearman's rank correlation, $p>0.9, R_{s}=$ $-0.0063)$. Thus, our normalized measure of attentional rate modulation is robust to differences in firing rate. As a final control, we compared the rate A.I. of the lower-firing rate bursty broad spiking neurons (mean unattended rate $<15 \mathrm{~Hz}, n=26$ ) with the rate A.I. of the higher-firing rate non-bursty broad spiking neurons (mean unattended rate $\geq 10 \mathrm{~Hz}, n=17$ ). As before, the bursty broad spiking neurons showed a significant increase in rate with attentional modulation (Wilcoxon's signed-rank test, $p<0.001$ ), whereas the non-bursty broad spiking neurons did not. Furthermore, the difference in attentional rate modulation between these groups remained significant (Mann-Whitney $U$ test, $p<0.01$ ).

We also repeated our analyses using an additional burstiness metric that was normalized by firing rate. Here, burstiness was measured as the mean of the autocorrelation function from 1-4 $\mathrm{ms}$ in unattended trials, divided by the mean firing rate in these trials. As before, we find that there is a significant correlation 

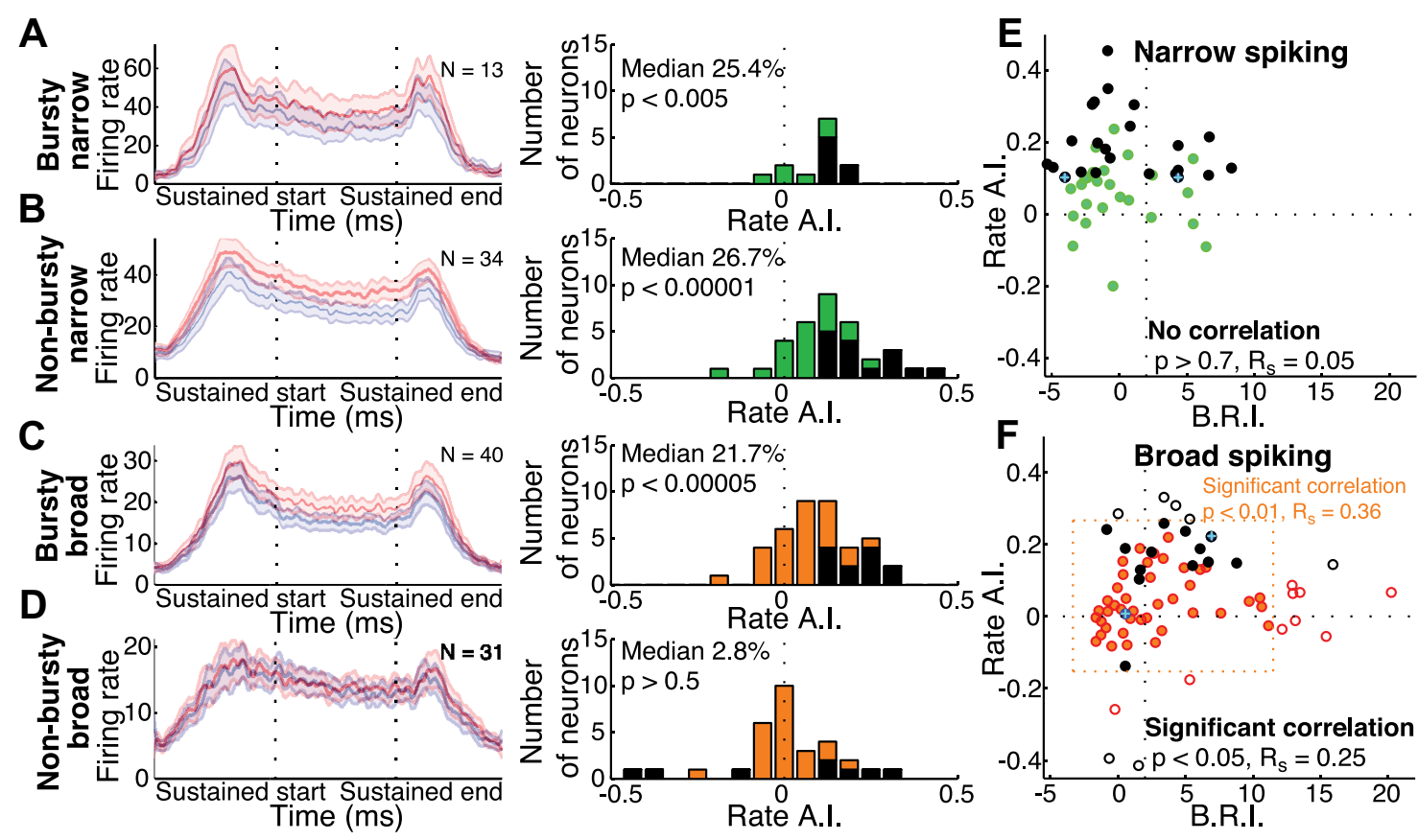

Figure 4. Relationship between burstiness/refractoriness index and attention-dependent rate modulation. $\boldsymbol{A}-\boldsymbol{D}$, Attention-dependent modulation of firing rate across four groups of V4 neurons: bursty narrow (A), non-bursty narrow (B), bursty broad ( $\boldsymbol{C}$, and non-bursty broad (D). Left columns show population mean stimulus-evoked responses for tracked (red traces) or ignored (blue traces) stimuli (data smoothed with a Gaussian filter in which $\sigma=25 \mathrm{~ms}$; shaded regions indicate \pm 1 SEM). The middle column shows the distributions of the firing rate attention index for each population, with individually significant units $(p<0.001)$ shaded black. $\boldsymbol{E}, \boldsymbol{F}$, Population scatter plots of B.R.I. versus firing rate A.I. Narrow spiking cells are shown in $\boldsymbol{E}$ (green circles), and broad spiking cells are shown in $\boldsymbol{F}$ (orange circles). Individual units with significant attention-dependent rate modulation $(p<0.001$ ) are shown in black. Points with blue crosses correspond to the example individual neurons in Figures 2 and 3 . In $F$, filled circles indicate broad spiking neurons with rate A.I. and B.R.I. values within $1.5 \mathrm{SD}$ of the broad spiking population mean (indicated by dashed orange box). There is a significant correlation between B.R.I. and rate A.I. across the entire broad spiking population (open and filled circles; Spearman's correlation, $p<0.05, R_{\mathrm{s}}=0.2499$ ) and for the subset within $1.5 \mathrm{SD}$ of the mean (filled circles; Spearman's correlation, $p<0.01, R_{\mathrm{s}}=0.3583$ ).

between burstiness and attention-dependent firing rate modulation among broad spiking (Spearman's rank correlation, $p<$ $\left.0.05, R_{\mathrm{s}}=0.2458\right)$ but not among narrow spiking neurons $(p>$ $\left.0.7, R_{\mathrm{s}}=-0.0458\right)$.

Session-to-session variability does not explain heterogeneity in attentional modulation or burstiness

We next considered whether the observed heterogeneity in attentional modulation could be accounted for by differences across sessions, perhaps reflecting differences in the internal state of the animal rather than differences in the intrinsic properties of individual neurons. We reasoned that, if the relationship between burstiness and attentional modulation stemmed from variation across sessions, then attentional modulation should be correlated across simultaneously recorded pairs of broad spiking neurons. However, we do not find a significant correlation in the rate A.I. pairs of broad spiking neurons $\left(n=52\right.$ pairs, $p>0.9, R_{s}=$ $-0.01)$. In fact, we see examples of pairs in which one neuron exhibited attention-dependent increases in firing rate, whereas the other exhibited reductions in rate. This pattern of results supports the conclusion that attentional modulation varies as a function of spike wave form and burstiness.

We find a related pattern of results for burstiness, with substantial variability in the degree of burstiness within the same session. In particular, we do not observe a significant correlation in the B.R.I. values of simultaneously recorded broad spiking neurons $\left(n=52\right.$ pairs, $\left.p>0.1, R_{\mathrm{s}}=0.12\right)$. We thus conclude that much of the heterogeneity we observe is attributable to differences in the neurons themselves, such as their intrinsic membrane properties or their place in the cortical circuit.

\section{Spontaneous burst firing correlated with stimulus-evoked burst firing}

A related question is whether the patterns we observed might perhaps reflect variations in stimulus conditions across experimental sessions. For example, neurons differ in contrast sensitivity, raising the possibility that differences in burstiness and attentional modulation might vary as a function of where the stimulus fell on the contrast response function of a given neuron. Such stimulus-dependent differences would not be expected to hold in the absence of a stimulus. Therefore, to test this possibility, we asked whether the burst firing properties observed during the stimulus-evoked response also held during the $250 \mathrm{~ms}$ spontaneous prestimulus period, when no stimulus of any kind was present. The low firing rates observed in this spontaneous period decreased our ability to accurately estimate the burst properties for some neurons. In particular, for a subset $(n=6)$ of neurons with very low spontaneous firing rates, the lack of action potentials led to artifactually high B.R.I values (B.R.I. $\gg 100$ ). We excluded these neurons from additional analysis. However, among the remaining neurons $(n=112)$, we found a strong correlation between the spontaneous period and sustained period B.R.I. estimates, both pooling across narrow and broad spiking neurons (Spearman's nonparametric correlation, $p \ll$ $\left.0.0001, R_{\mathrm{s}}=0.81\right)$ as well as among the narrow and broad spiking subpopulations (broad, $p \ll 0.0001, R_{\mathrm{s}}=0.79$; narrow, $p \ll$ $\left.0.0001, R_{\mathrm{s}}=0.70\right)$. This suggests that the degree of burstiness in our neuronal population is not an artifact of the particular experimental conditions in an individual recording session but instead reflects properties intrinsic to the neuron, either attributable to 

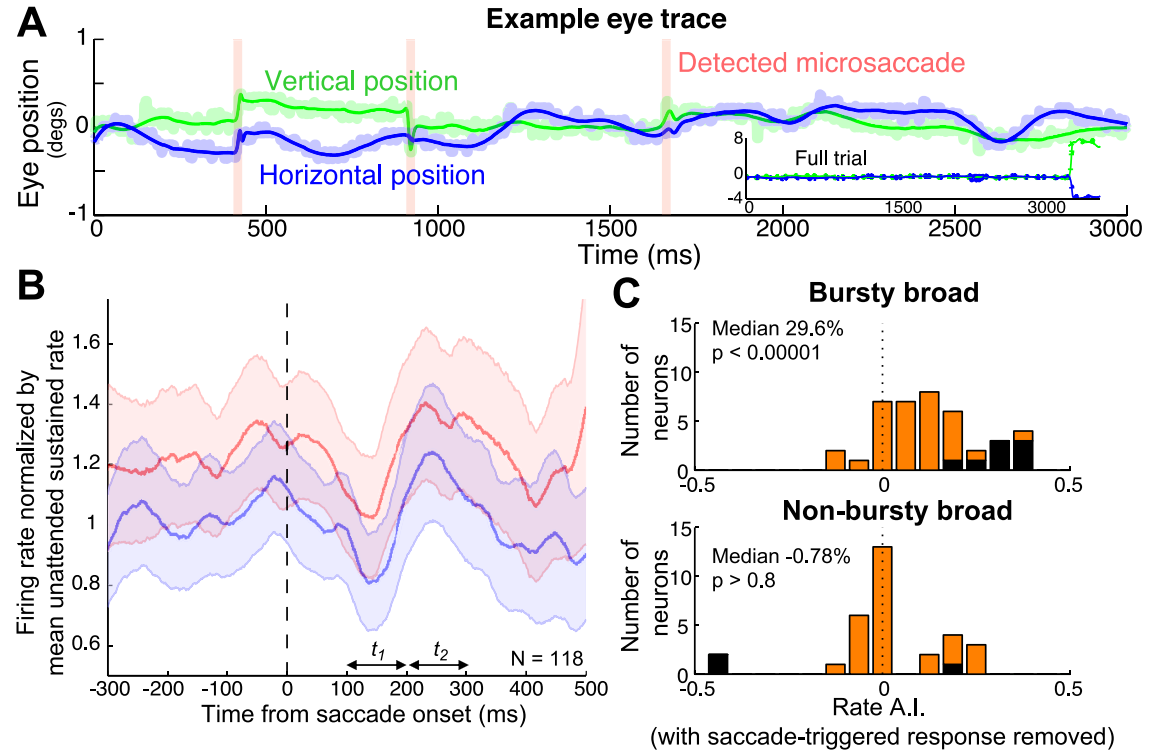

Figure 5. Microsaccade-triggered response modulation. $\boldsymbol{A}$, Single-trial example of the saccade detection algorithm (see Materials and Methods). Blue and green curves correspond to the model fits of the horizontal and vertical eye position, with shaded lines indicating the raw position traces. Detected microsaccades are indicated by pink vertical bars. Inset shows eye position across the entire trial (including the saccade to the cued stimulus at the end of the trial). $\boldsymbol{B}$, Microsaccade-triggered responses averaged across the entire population. Curves show the population mean of normalized microsaccade-triggered responses to when the stimulus was tracked (red traces) or ignored (blue). Data are smoothed with a Gaussian filter in which $\sigma=25 \mathrm{~ms}$; shaded regions indicate \pm 1 SEM. C, Distribution of the firing rate attention index for the bursty and non-bursty broad spiking groups, calculated after removing all action potentials that occurred within $400 \mathrm{~ms}$ after a detected microsaccade. Individually significant units $(p<0.001)$ are shaded black.

intrinsic neuronal properties such as membrane conductance or perhaps to the position of the neuron within the cortical circuit.

If burstiness is a neuron property that predicts attentional modulation, we reasoned that we might see a systematic relationship between burstiness during the spontaneous period and attentional rate modulation during the stimulus-evoked response period. To examine this, we first subdivided the broad spiking neurons, based on their spontaneous activity, into bursty and non-bursty groups using the same burst metric and definition of burstiness we previously applied to the stimulus-evoked response. That is, we classified cells as bursty or non-bursty according to whether the B.R.I. derived from spontaneous activity exceeded the $2 \mathrm{SD}$ threshold. As before, we found significant attentional modulation among bursty broad spiking neurons (Wilcoxon's signed-rank test, $n=39, p<0.00005$ ) but not among non-bursty broad spiking neurons $(n=27, p>0.2)$. Furthermore, the difference in attentional modulation between these two groups remained significant (Mann-Whitney $U$ test, $p=0.005)$. We also tested whether there was a significant correlation between the spontaneous B.R.I. and the rate A.I. of the sustained period. As before (Fig. $4 F$ ), we excluded outliers falling outside of a $1.5 \mathrm{SD}$ window around the population mean B.R.I. and rate A.I. Despite the loss of statistical power inherent in the lower spike rates observed in the prestimulus period, there was, among broad spiking neurons, a significant correlation between the spontaneous level of burstiness and attentional modulation $\left(p<0.02, R_{\mathrm{s}}=0.3351\right)$.

\section{Eye movements do not explain differences in attentional modulation}

Previous studies have found that even small eye movements, such as fixational microsaccades, can influence neuronal responses (Gur et al., 1997; Martinez-Conde et al., 2004; Gur and Snod- derly, 2006). We therefore examined whether differences in eye movements across the bursty and non-bursty broad and narrow spiking groups could underlie the differences in attention effects we observe. We used a saccade detection algorithm to identify microsaccades within the sustained response period (see Materials and Methods). Figure $5 A$ shows microsaccades detected by the algorithm on a single trial. Green and blue lines show, respectively, fits to vertical and horizontal eye position, and three detected microsaccades are indicated by pink vertical bars.

To examine the impact of microsaccades on neural responses, we computed the mean microsaccade-triggered response, normalized on a neuron-by-neuron basis to the mean response evoked by the ignored stimulus. Figure $5 B$ shows the mean microsaccade-triggered response across the entire population computed for attended (red) and unattended (blue) trials. As expected, the firing rates are higher, on average, in the attended condition, but there is no obvious difference in the deviations of the response that were induced by the microsaccade. In both conditions, the microsaccade-triggered response initially shows a transient reduction in firing rate followed by a brief increase in firing. This response is similar to that reported by Mitchell et al. (2007) but is somewhat smaller in amplitude and delayed in time compared with the V4 responses observed by Leopold and Logothetis (1998). To quantify the saccade-induced response across the population, we computed a microsaccadetriggered response index (M.T.R.I.), which we define as the ratio between the mean normalized microsaccade-triggered response 200-300 and 100-200 ms after a detected microsaccade. We find that the M.T.R.I. during the presentation of an unattended stimulus in the receptive field is significantly greater than unity across the entire population and for each of bursty and non-bursty broad and narrow spiking groups, indicating that the responses of all four subclasses of neurons were modulated by microsaccades (Wilcoxon's signed-rank test, entire population, $p<0.00000001$; bursty broad, $p<0.005$; non-bursty broad, $p<0.0005$; bursty-narrow, $p<0.05$; non-bursty narrow, $p<0.005$ ).

Given that we see a significant modulation in the firing rate response after microsaccades, we next examined whether differences in eye movements could account for the differences we observe between the bursty and non-bursty broad and narrow spiking groups. First, we tested whether the number, size, direction, or peak velocity of microsaccades varied between the nonbursty broad group and any of the other three groups in either attention condition. It did not differ significantly in any of these measures (Mann-Whitney $U$ test, all $p$ values $>0.1$ ). We next examined whether there was a correlation between the B.R.I. and the number, size, direction, or peak velocity of microsaccades in either attention condition. We find no correlation, either across the entire population or across broad spiking cells (Spearman's rank correlation, all $p$ values $>0.05$ ). Finally, we tested whether there was a difference in the magnitude of the unattended M.T.R.I. across the four groups. There was no significant difference (Mann-Whitney $U$ test, all $p$ values $>0.5$ ). 
As an additional test, we repeated our burst and attention analyses after removing all action potentials that occurred within $400 \mathrm{~ms}$ after each detected microsaccade. This did not significantly change any of our findings. This is shown in Figure $5 C$, which shows the distribution of the firing rate attention index among bursty (above) and non-bursty broad spiking neurons (below), calculated after removing the action potentials immediately after microsaccades. As was the case previously, we find that attention induced a significant attention-dependent increase in firing rate among the bursty broad spiking neurons (Wilcoxon's signed-rank test, $p<0.00001)$ and among the narrow spiking neurons (bursty narrow, $p<0.05$; non-bursty narrow, $p<$ 0.00001; data not shown) but not among the non-bursty broad spiking neurons $(p>0.8)$. Furthermore, attentional modulation among non-bursty broad spiking neurons remained significantly different from the increase in firing rate with attention seen among the bursty broad spiking group (Mann-Whitney $U$ test, $p<0.005)$. We also found that the correlation between B.R.I. and rate A.I. among the broad spiking neurons remained significant (Spearman's nonparametric correlation, $p<0.05, R_{\mathrm{s}}=0.2530$ ). Thus, the differences in attentional modulation between bursty and non-bursty broad spiking neurons we see cannot be attributed to eye movements.

\section{Discussion}

The primary goals of this study were to better characterize an aspect of response heterogeneity in macaque area V4 - burst firing - and determine whether this heterogeneity could help account for variability in attention-dependent rate modulation. We observed considerable variability in burst firing across the population, spanning the gamut from neurons that fire in a manner consistent with a refractory-limited Poisson process to neurons that fire numerous bursts of action potentials. The range of burstiness was particularly pronounced among broad spiking neurons, which were, on average, more bursty than narrow spiking neurons and which included the most bursty neurons in our population. This is similar to observations in prefrontal and parietal areas by Compte et al. (2003) and Constantinidis and Goldman-Rakic (2002), who report that many of their narrow spiking cells had pronounced relative refractory periods and found that almost all of their highly bursty cells were broad spiking cells.

Our second main finding is that attentional modulation varied as a function of burstiness. Here, we found a clear difference between narrow and broad spiking neurons. There was no evidence for a correlation between burstiness and attentional modulation among narrow spiking neurons and no significant difference in attentional modulation for narrow spiking neurons classified as bursty versus non-bursty. In contrast, among broad spiking neurons, there is a significant positive correlation between the degree of burstiness and the magnitude of attentiondependent rate modulation. Furthermore, among bursty broad spiking neurons, attention caused a significant median increase in firing rate $(21.7 \%)$ compared with a nonsignificant change (2.8\%) among non-bursty broad spiking neurons. Among bursty broad spiking neurons, all neurons showing individually significant rate modulation with attention showed increases in mean rate, whereas among non-bursty broad spiking neurons, attention caused both increases and decreases in mean rate. Across our population, significant decreases in firing rate with attention were thus restricted to non-bursty broad spiking neurons. These results therefore lead to the conclusion that variation in the degree of burstiness helps account for the wider range of attentional modulation among broad spiking neurons compared with narrow spiking neurons (Mitchell et al., 2007).

\section{Firing rates}

There are several potential confounds that could have contributed to the differences in attention-dependent rate modulation we observe between the bursty and non-bursty broad spiking neurons. In particular, if the bursty broad spiking neurons had higher firing rates than the non-bursty broad spiking neurons, our ability to detect attentional modulation among the nonbursty broad spiking neurons could have been impaired relative to the bursty broad spiking neurons. We ruled this out by conducting control analyses in which we compared responses evoked by unattended stimuli. We found no significant difference in median firing rate between these two groups. Furthermore, when we compare high firing rate non-bursty neurons with low firing rate bursty neurons, we continued to see significant differences in attentional modulation between these groups. Thus, we conclude that the differences we observe are not a rate artifact.

\section{Threshold for burstiness}

A second potential concern was that the differences we see between bursty and non-bursty broad spiking cells might potentially have depended on the particular threshold we used to define the boundary between these two groups. We addressed this by examining the data without dividing neurons into bursty and non-bursty groups. In this analysis, we tested whether there is a significant correlation between the degree of burstiness and attention-dependent firing rate modulation. We found that, among broad spiking neurons, there was a significant positive correlation between burstiness and attention-dependent increases in firing rate. Thus, attentional modulation increased as a function of burstiness, independent of the boundary we set between bursty and non-bursty neurons. We also validated this by repeating the analysis using a different burstiness metric that was normalized by mean firing rate. Again, there was a significant correlation for broad spiking neurons but not for narrow spiking neurons.

\section{Eye movements}

The final possible confound we ruled out was whether there were differences in eye movements or in eye-movement-triggered responses that could underlie the differences in attentional modulation we observed. These possibilities struck us as unlikely in light of the fact that many of the bursty and non-bursty neurons were recorded simultaneously and were therefore subject to identical eye movements. However, to address this directly, we determined that there were no differences in the number, direction, or velocity of microsaccades across broad spiking and narrow spiking neurons. As a final control, we reanalyzed the data after removing spikes recorded after microsaccades. The relationship between burst firing and attention-dependent rate modulation remained significant.

\section{The relationship between attentional modulation and burstiness reflects intrinsic cell properties}

Previous studies have found that attentional modulation can vary markedly as a function of stimulus conditions, such as where the stimulus falls along the contrast response function of a neuron (Reynolds et al., 2000; Reynolds and Chelazzi, 2004; Williford and Maunsell, 2006; Reynolds and Heeger, 2009). Variation in these factors may have contributed to the heterogeneity of burst firing and attentional modulation we observed. However, the 
heterogeneity we observe is preserved across stimulus conditions, suggesting that it is attributable to intrinsic cell properties. In particular, we found that burstiness in the stimulus-evoked period was very strongly correlated with burstiness in the prestimulus period, when no stimuli were present. Furthermore, the degree of burstiness during this prestimulus period was correlated with attentional modulation of firing rate during the later stimulus-evoked response period. Therefore, we conclude that differences in stimulus-evoked response properties cannot account for the present results.

We find a related pattern of results for burstiness, with substantial variability in the degree of burstiness within the same session. B.R.I. values of simultaneously recorded broad spiking neurons were not correlated with one another $(n=52$ pairs, $p>$ $0.1, R_{\mathrm{s}}=0.12$ ). We thus conclude that much of the heterogeneity we observe is attributable to differences in the neurons themselves, such as their intrinsic membrane properties or their place in the cortical circuit.

\section{Potential anatomical correlates}

Given the differences in attentional modulation between bursty and non-bursty broad spiking neurons, it is tempting to speculate that these may correspond to anatomically distinct classes of neurons. Intracellular recording studies have used burstiness as one of the key metrics to distinguish among classes of neurons, including "intrinsically bursting" neurons, pyramidal neurons that emit short bursts of action potentials (McCormick et al., 1985; Connors and Gutnick, 1990). A substantial portion of these neurons occupy layer $\mathrm{V}$ and project to the superior colliculus and the pontine nucleus (Agmon and Connors, 1992; Wang and McCormick, 1993; Kasper et al., 1994). These can be distinguished from another class of bursting neurons, called "chattering cells" or "fast rhythmic bursting" cells, which have been found in layers II/III of ferret and cat cortex and which exhibit narrow action potentials (Brumberg et al., 2000; Nowak et al., 2003). Although less common in our dataset, we did observe some narrow spiking neurons with significant burst firing. These should be distinguished from narrow spiking neurons that lack burst firing, which are instead likely to correspond to fast-spiking interneurons. One recent study (Katai et al., 2010) has used spiking statistics to distinguish among four different cell classes in the frontal cortex of the behaving primate. Their study identified these with classes previously defined in slice (intrinsically bursting, regular spiking, fast spiking, and fast rhythmic bursting). However, we do not find evidence, in V4, for distinct classes of bursting and non-bursting neurons. The range of bursting behavior in V4 appears to fall along a continuum, as has also been seen in middle temporal area MT (Bair et al., 1994). Therefore, although variability in burst firing can help account for variability in attentional modulation, our data do not allow us to conclude that the bursty broad spiking neurons we find modulated by attention constitute a distinct class of neurons.

Nonetheless, it would be of significant value to determine whether bursty and non-bursty broad spiking neurons recorded in the awake primate do, in fact, correspond to anatomically distinct classes of neurons. If they do, understanding where these neurons fit into the local cortical circuit could lead to major new insights into the role of attentional modulation of visual cortical neurons. If, for example, the bursty broad spiking neurons that we find are modulated by attention correspond to layer $\mathrm{V}$ intrinsically bursty neurons, this would, by virtue of their corticotectal projection patterns, implicate attentional modulation of area $\mathrm{V} 4$ neurons as playing a role in modulating sensory input to the oculomotor system rather than the more traditional view that attention serves to modulate ascending sensory signals as they progress from V4 to higher-order visual areas. Furthermore, because layer $\mathrm{V}$ corticotectal neurons correspond to tall-tufted pyramidal neurons, this would suggest that the laminar distribution of attentional feedback signals are directed toward the layers from which these pyramidal neurons receive input (Kasper et al., 1994; Larsen et al., 2007).

\section{References}

Agmon A, Connors BW (1992) Correlation between intrinsic firing patterns and thalamocortical synaptic responses of neurons in mouse barrel cortex. J Neurosci 12:319-329.

Bair W, Koch C, Newsome W, Britten K (1994) Power spectrum analysis of bursting cells in are MT in the behaving monkey. J Neurosci 14:2870-2892.

Barthó P, Hirase H, Monconduit L, Zugaro M, Harris KD, Buzsáki G (2004) Characterization of neocortical principal cells and interneurons by network interactions and extracellular features. J Neurophysiol 92:600-608.

Brumberg JC, Nowak LG, McCormick DA (2000) Ionic mechanisms underlying repetitive high-frequency burst firing in supragranular cortical neurons. J Neurosci 20:4829-4843.

Cavanagh P, Alvarez GA (2005) Tracking multiple targets with multifocal attention. Trends Cogn Sci 9:349-354.

Compte A, Constantinidis C, Tegner J, Raghavachari S, Chafee MV, Goldman-Rakic PS, Wang XJ (2003) Temporally irregular mnemonic persistent activity in prefrontal neurons of monkeys during a delayed response task. J Neurophysiol 90:3441-3454

Connors BW, Gutnick MJ (1990) Intrinsic firing patterns of diverse neocortical neurons. Trends Neurosci 13:99-104.

Constantinidis C, Goldman-Rakic PS (2002) Correlated discharges among putative pyramidal neurons and interneurons in primate prefrontal cortex. J Neurophysiol 88:3487-3497.

Friedman-Hill S, Maldonado PE, Gray CM (2000) Dynamics of striate cortical activity in the alert macaque. I. Incidence and stimulus-dependence of gamma-band neuronal oscillations. Cereb Cortex 10:1105-1116.

Gur M, Snodderly DM (2006) High response reliability of neurons in primary visual cortex (V1) of alert, trained monkeys. Cereb Cortex 16:888-895.

Gur M, Beylin A, Snodderly DM (1997) Response variability of neurons in primary visual cortex (V1) of alert monkeys. J Neurosci 17:2914-2920.

Joelving FC, Compte A, Constantinidis C (2007) Temporal properties of posterior parietal neuron discharges during working memory and passive viewing. J Neurophysiol 97:2254-2266.

Kasper EM, Larkman AU, Lübke J, Blakemore C (1994) Pyramidal neurons in layer 5 of the rat visual cortex. I. Correlation among cell morphology, intrinsic electrophysiological properties, and axon targets. J Comp Neurol 339:459-474.

Katai S, Kato K, Unno S, Kang Y, Saruwatari M, Ishikawa N, Inoue M, Mikami A (2010) Classification of extracellularly recorded neurons by their discharge patterns and their correlates with intracellularly identified neuronal types in the frontal cortex of behaving monkeys. Eur J Neurosci 31:1322-1338.

Knudsen EI (2007) Fundamental components of attention. Annu Rev Neurosci 30:57-78.

Larsen DD, Wickersham IR, Callaway EM (2007) Retrograde tracing with recombinant rabies virus reveals correlations between projection targets and dendritic architecture in layer 5 of mouse barrel cortex. Front Neural Circuits 1:5.

Leopold DA, Logothetis NK (1998) Microsaccades differentially modulate neural activity in the striate and extrastriate visual cortex. Exp Brain Res 123:341-345.

Maimon G, Assad JA (2009) Beyond Poisson: increased spike-time regularity across primate parietal cortex. Neuron 62:426-440.

Martinez-Conde S, Macknik SL, Hubel DH (2004) The role of fixational eye movements in visual perception. Nat Rev Neurosci 5:229-240.

McCormick DA, Connors BW, Lighthall JW, Prince DA (1985) Comparative electrophysiology of pyramidal and sparsely spiny neurons of the neocortex. J Neurophysiol 54:782-806.

Mitchell JF, Sundberg KA, Reynolds JH (2007) Differential attentiondependent response modulation across cell classes in macaque visual area V4. Neuron 55:131-141. 
Mitchell JF, Sundberg KA, Reynolds JH (2009) Spatial attention decorrelates intrinsic activity fluctuations in macaque area V4. Neuron 63:879-888.

Nowak LG, Azouz R, Sanchez-Vives MV, Gray CM, McCormick DA (2003) Electrophysiological classes of cat primary visual cortical neurons in vivo as revealed by quantitative analyses. J Neurophysiol 89:1541-1566.

Pylyshyn ZW, Storm RW (1988) Tracking multiple independent targets: evidence for a parallel tracking mechanism. Spat Vis 3:179-197.

Reynolds JH, Chelazzi L (2004) Attentional modulation of visual processing. Annu Rev Neurosci 27:611-647.

Reynolds JH, Heeger DJ (2009) The normalization model of attention. Neuron 61:168-185.

Reynolds JH, Pasternak T, Desimone R (2000) Attention increases sensitivity of V4 neurons. Neuron 26:703-714.

Ringach DL, Hawken MJ, Shapley R (1997) Dynamics of orientation tuning in macaque primary visual cortex. Nature 387:281-284.

Sears CR, Pylyshyn ZW (2000) Multiple object tracking and attentional processing. Can J Exp Psychol 54:1-14.

Shadlen MN, Newsome WT (1998) The variable discharge of cortical neu- rons: implications for connectivity, computation, and information coding. J Neurosci 18:3870-3896.

Softky WR, Koch C (1993) The highly irregular firing of cortical cells is inconsistent with temporal integration of random EPSPs. J Neurosci 13:334-350.

Sundberg KA, Mitchell JF, Reynolds JH (2009) Spatial attention modulates center-surround interactions in macaque visual area V4. Neuron 61:952-963.

Tolhurst DJ, Movshon JA, Dean AF (1983) The statistical reliability of signals in single neurons in cat and monkey visual cortex. Vision Res 23:775-785.

Treue S (2003) Visual attention: the where, what, how and why of saliency. Curr Opin Neurobiol 13:428-432.

Wang Z, McCormick DA (1993) Control of firing mode of corticotectal and corticopontine layer $\mathrm{V}$ burst-generating neurons by norepinephrine, acetylcholine, and 1S,3R-ACPD. J Neurosci 13:2199-2216.

Williford T, Maunsell JH (2006) Effects of spatial attention on contrast response functions in macaque area V4. J Neurophysiol 96:40-54. 